\title{
Close Relationship Between Sympathetic Activation and Coronary Microvascular Dysfunction During Acute Hyperglycemia in Subjects With Atherosclerotic Risk Factors
}

\author{
Yasuyoshi Takei, MD; Hirofumi Tomiyama, MD; \\ Nobuhiro Tanaka, MD; Akira Yamashina, MD
}

\begin{abstract}
Background The effect of acute hyperglycemia (AHG) during the oral glucose tolerance test (OGTT) on coronary microvascular function was evaluated, as well as the associations among the changes in coronary microvascular function, oxidative stress, and sympathetic tone.

Methods and Results Transthoracic Doppler echocardiography and OGTT were performed in 24 subjects with atherosclerotic risk factors ( $61 \pm 9$ years). The coronary flow velocity before and during the infusion of adenosine (CFVhyp), plasma levels of thiobarbituric acid-reactive substances (TBARS), and the low-frequency/highfrequency power (LF/HF) ratio yielded by power spectral analysis of heart rate variability were measured before and at $1 \mathrm{~h}$ during 75-g OGTT. AHG significantly decreased the CFVhyp, and increased the TBARS and LF/HF. Multiple linear regression analysis revealed that the percent changes in the CFVhyp were significantly associated with the percent changes in the $L F / H F$ ratio $\beta=-0.43, \mathrm{p}<0.05$ ).

Conclusion In subjects with atherosclerotic risk factors who may be considered likely to have atherosclerotic arterial damage, AHG seems to induce concomitant coronary microvascular dysfunction, increased oxidative stress, and sympathetic activation. Coronary microvascular dysfunction, therefore, appears to be closely related to sympathetic activation. (Circ J 2007; 71: 202-206)
\end{abstract}

Key Words: Coronary circulation; Hyperglycemia; Oxidative stress; Sympathetic nervous system

$\mathbf{T}$ he Framingham offspring study has demonstrated that post-challenge hyperglycemia is an independent risk factor for cardiovascular disease, and that stress hyperglycemia is one of the determinants of the outcome after myocardial infarction.,3 In this context, it is thought that coronary microvascular dysfunction related to stress hyperglycemia might be one of the possible reasons for the poor outcome in patients with acute myocardial infarction with stress hyperglycemia, and also for the improvement of epicardial coronary blood flow and outcome in these patients after nicorandil administration. Thus, the association between acute hyperglycemia (AHG) and coronary microvascular function (ie, hyperemic response of coronary blood flow or coronary flow reserve) should be thoroughly examined. Although some studies have demonstrated that AHG may impair flow-mediated vasodilatation in the peripheral arteries 5,6 which might be a predictor of future cardiovascular events? the effect of AHG on coronary microvascular function has not been fully clarified. Increased oxidative stress ${ }^{8}$ and/or sympathetic activation ${ }^{9}$ have been demonstrated to contribute to impairment of coronary microvascular function, and both of these pathophysiological abnormalities are also observed during AHG.

(Received June 28, 2006; revised manuscript received September 12, 2006; accepted November 9, 2006)

Second Department of Internal Medicine, Tokyo Medical University, Tokyo, Japan

Mailing address: Hirofumi Tomiyama, MD, Second Department of Internal Medicine, Tokyo Medical University, 6-7-1 Nishi-shinjuku, Shinjuku-ku, Tokyo 160-0023, Japan
However, the influence of these abnormalities caused by AHG on coronary microvascular function has not been fully evaluated.

Recently, transthoracic Doppler, which can be used to estimate coronary microvascular function in a non-invasive manner, has become feasible in the clinical setting!10-12 Therefore, we chose to use it in the present study to evaluate the effects of AHG observed during the oral glucose tolerance test (OGTT) on coronary microvascular function, and the associations among changes in the hyperemic response of coronary blood flow velocity (CFV) or coronary flow velocity reserve (CFVR), oxidative stress, and increased sympathetic tone caused by AHG in subjects with atherosclerotic risk factors.

\section{Methods}

Subjects

Twenty-four patients receiving treatment for atherosclerotic risk factors (14 patients with hypertension, 6 with hypercholesterolemia, 3 with hypertension and hypercholesterolemia, and 1 patient with abnormal glucose intolerance) at Tokyo Medical University Hospital were enrolled in this study. None of the patients had any symptoms suggestive of coronary heart disease or changes on the electrocardiogram suggestive of myocardial ischemia. Furthermore, none of them showed any regional wall motion abnormality on echocardiography. Written informed consent was given by all the subjects prior to their participation and the study protocol was approved by the Ethics Com- 
mittee of Tokyo Medical University.

\section{Study Protocol}

The examination was started at $09.00 \mathrm{~h}$ after the subjects had fasted for at least $8 \mathrm{~h}$. Prescribed medications were withheld on the day of the experiment. The measurements were performed in a quiet room with temperature adjusted to $23-25^{\circ} \mathrm{C}$. The subjects were instructed to abstain from smoking for at least $24 \mathrm{~h}$ prior to the start of the study. At the start of the study, just prior to oral glucose administration to induce hyperglycemia, measurement of heart rate variability (HRV) and assessment of coronary microvascular function (measurement of CFV in the distal left anterior descending coronary artery (LAD) by transthoracic echocardiography at baseline and during hyperemia) were conducted. Blood pressure and heart rate were monitored every minute during the echocardiographic measurement of CFV. Then, the subjects were given an oral glucose load of $75 \mathrm{~g}$ (Torelan G, Takeda Pharmaceutical Company Limited, Tokyo, Japan; 75-g OGTT) and $1 \mathrm{~h}$ later measurement of HRV and assessment of coronary microvascular function by transthoracic echocardiography were repeated.

\section{Measurement of CFVR by Transthoracic Doppler Echocardiography}

The Sequoia 512 (SIEMENS AG equipment) was used as the ultrasonography equipment in combination with a $3.5-7.0 \mathrm{MHz}$ broadband probe. After examination of left ventricular wall motion, left ventricular diameter, left ventricular systolic function, and left ventricular diastolic function, the subject was asked to lie in the left semilateral position, and the broadband probe was placed over the 4 th $/ 5$ th intercostal space in the left midclavicular line to detect blood flow in the distal segment of the LAD from an apical approach, guided by color Doppler imaging. The color Doppler velocity was set in the range of $6.9-32 \mathrm{~cm} / \mathrm{s}$. After the coronary blood flow signals were confirmed, the CFV was measured by pulsed Doppler method to determine the mean diastolic CFV. The Doppler sample volume was $2 \mathrm{~mm}$ in width, and the Doppler angle correction was less than $30^{\circ}$ (mean $22 \pm 6^{\circ}$ ). By positioning a sample volume on the color signal in the distal segment of the LAD, Doppler spectral tracings of flow velocity were recorded by fast Fourier transform analysis. The spectral Doppler signals of the flow in the LAD showed a characteristic biphasic pattern with a larger diastolic component and smaller systolic one. Measurements were performed by tracing the contour of the spectral Doppler signals using the computer incorporated in the ultrasound system. After measurement of the $\mathrm{CFV}$ at baseline ( $\mathrm{CFV}$ base), the hyperemic mean diastolic CFV (CFVhyp) induced by intravenous administration of adenosine triphosphate $\left(0.15 \mathrm{mg} \cdot \mathrm{kg}^{-1} \cdot \mathrm{min}^{-1}\right)$ was measured ${ }^{10,11} \mathrm{CFV}$ base and $\mathrm{CFVhyp}$ were determined as the mean of the values obtained during 3 consecutive heart beats, and the ratio between the 2 values was calculated as the CFVR. The recorded waveforms were saved on s-VHS video tapes and magneto-optical disk as digital data for subsequent off-line analysis. The acceptable reproducibility of CFVR has been reported before! ${ }^{12,13}$ In addition, the reproducibility of each of the measurements at our institute is as follows: in 45 volunteers, there was good agreement between 2 observers' measurements for CFV base, CFVhyp, and CFVR ( $r=0.99, r=0.96$, and $r=0.96$, respectively). Interobserver and intraobserver variabilities for the measurement of the Doppler velocity were $4.8 \%$ and $4.1 \%$, respectively.

\section{Laboratory Measurements}

Before the start of the 75-g OGTT, a fasting blood sample was collected from the subjects, and the following parameters were measured using standard techniques: plasma levels of glucose, insulin, total cholesterol, highdensity lipoprotein-cholesterol, low-density lipoproteincholesterol, and triglycerides. Blood samples were also collected to measure blood glucose and plasma insulin levels at 30,60, and $120 \mathrm{~min}$ after the oral glucose load. The plasma level of thiobarbituric acid-reactive substances (TBARS) was used as a marker of oxidative stress. Briefly, lipid peroxidation is one of the major consequences of free radical-mediated injury. Initially lipid peroxidation leads to the production of conjugated dienic hydroperoxides. These unstable substances decompose into TBARS. The TBARS are measured by a spectrophotometric assay that quantifies a chromogen produced by the reaction of thiobarbituric acid with malondialdehyde!5,16 Therefore, in the present study TBARS were determined before and $1 \mathrm{~h}$ after the oral glucose load. For this measurement, plasma was mixed with $2 \%$ butylated hydroxytoluene and the Quintanilla reagent $(26 \mathrm{mmol} / \mathrm{L}$ thiobarbituric acid and $918 \mathrm{mmol} / \mathrm{L}$ trichloroacetic acid). The mixture was boiled for $15 \mathrm{~min}$ to facilitate the reactions, then the reaction mixture was cooled and centrifuged at $3,000 \mathrm{G}$ for $10 \mathrm{~min}$. The soluble phase was measured by spectrophotometry at a wavelength of $535 \mathrm{~nm}$.

\section{Measurement of HRV}

Each subject underwent ECG recording after they had rested in the supine position for $10 \mathrm{~min}$ in a quiet room in which the temperature was adjusted to $23-25^{\circ} \mathrm{C}$. The ECG $\mathrm{R}-\mathrm{R}$ interval data obtained from the $\mathrm{CM} 5$ lead were then digitized at $1,000 \mathrm{~Hz}$, and the derived $\mathrm{R}-\mathrm{R}$ interval time series were aligned in a $2-\mathrm{Hz}$ sequence for power spectral analysis. The data were collected for $5 \mathrm{~min}$ of breath-in and breath-out at a frequency of $0.25 \mathrm{~Hz}$. Spectral analysis was performed with the discrete Fourier transform algorithm. Power spectra were quantified by measuring the areas under the 2 frequency bandwidths: $0.04-0.15 \mathrm{~Hz}$ (low-frequency power, LF) and $0.15-0.40 \mathrm{~Hz}$ (high-frequency power, HF), using the maximum entropy method (MemCalc; Suwa Trust, Tokyo, Japan)! ${ }^{17-19}$ The ratio of the LF/HF was calculated and used as a marker of sympathetic tone! ${ }^{1-19}$

\section{Statistical Analysis}

All data are expressed as mean \pm SD. The differences in the magnitude of each of the variables between before and $1 \mathrm{~h}$ after the oral glucose load in the 75-g OGTT were evaluated using a paired t-test. The percent changes in CFVhyp, CFVR, LF/HF and TBARS were then calculated as the values at $1 \mathrm{~h}$ after the oral glucose load in the 75-g OGTT: values before the start of the 75 -g OGTT) $\times 100 /$ the values before the start of the 75-g OGTT. The relationships among the changes in the variables were assessed by linear regression analysis. Next, the independencies of the relationships were assessed by step-wised multiple linear regression analysis adjusted for age, gender, smoking status, body mass index, 75-g OGTT status (ie, normal glucose tolerance/impaired glucose tolerance/diabetes $)^{20}$ medication (angiotensin-converting enzyme inhibitors, angiotensin II receptor blockers, calcium-channel antagonists, $\beta$-blockers, and statins), and the percent changes in the pressure-rate product. All the analyses were conducted using SPSS software for Windows, version 11.0J (SPSS, Chicago, IL, USA) and p-values of $<0.05$ were considered to be statisti- 
Table 1 Clinical Characteristics of the Subjects

\begin{tabular}{|c|c|}
\hline Age (years) & $61 \pm 9$ \\
\hline Gender $(M / F)$ & $19 / 5$ \\
\hline$B M I$ & $24.5 \pm 3.5$ \\
\hline Smoking (n) & 13 \\
\hline TC $(\mathrm{mmol} / \mathrm{L})$ & $5.3 \pm 0.9$ \\
\hline$H D L-C(\mathrm{mmol} / \mathrm{L})$ & $1.4 \pm 0.5$ \\
\hline$T G(\mathrm{mmol} / \mathrm{L})$ & $1.5 \pm 0.6$ \\
\hline Fasting plasma glucose $(\mathrm{mmol} / \mathrm{L})$ & $5.4 \pm 0.5$ \\
\hline Plasma glucose OGTTın $(\mathrm{mmol} / \mathrm{L})$ & $10.4 \pm 2.3$ \\
\hline Fasting plasma insulin (IU/ml) & $8.1 \pm 4.1$ \\
\hline Plasma insulin OGTTIh $(I U / m l)$ & $57.3 \pm 33.6$ \\
\hline \multicolumn{2}{|l|}{ Medication (n) } \\
\hline$A C E I$ & 1 \\
\hline Angiotensin II receptor blocker & 4 \\
\hline Calcium-channel antagonist & 10 \\
\hline$\beta$-blocker & 2 \\
\hline Statin & 9 \\
\hline
\end{tabular}

BMI, body mass index; TC, total cholesterol; HDL-C, high-density lipoprotein-cholesterol; TG, triglycerides; OGTTh, value obtained at $1 \mathrm{~h}$ in the oral glucose tolerance test; ACEI, angiotensin converting enzyme inhibitor.
Table 2 Changes in Parameters Before and During Acute Hyperglycemia

\begin{tabular}{|c|c|c|c|}
\hline & Before & OGTTlh $_{1}$ & $p$-value \\
\hline$S B P(m m H g)$ & $128 \pm 16$ & $129 \pm 13$ & $N S$ \\
\hline$D B P(m m H g)$ & $75 \pm 13$ & $76 \pm 10$ & $N S$ \\
\hline$H R$ (beats/min) & $57 \pm 8$ & $60 \pm 12$ & $<0.05$ \\
\hline$P R P$ & $7,353 \pm 1,465$ & $7,657 \pm 1,383$ & $<0.05$ \\
\hline$L F\left(\ln m s^{2}\right)$ & $3.6 \pm 3.7$ & $5.3 \pm 5.7$ & $<0.05$ \\
\hline$H F\left(\ln m s^{2}\right)$ & $3.7 \pm 3.5$ & $5.1 \pm 5.2$ & $N S$ \\
\hline$L F / H F$ & $1.4 \pm 1.0$ & $1.8 \pm 1.6$ & $<0.05$ \\
\hline TBARS $(\mathrm{nmol} / \mathrm{ml})$ & $2.6 \pm 0.6$ & $2.8 \pm 0.8$ & $<0.01$ \\
\hline$C F V_{\text {base }}(\mathrm{cm} / \mathrm{s})$ & $22.2 \pm 3.3$ & $25.1 \pm 5.6$ & $<0.01$ \\
\hline$C F V$ hyp $(\mathrm{cm} / \mathrm{s})$ & $72.1 \pm 18.4$ & $65.2 \pm 16.5$ & $<0.01$ \\
\hline$C F V R$ & $3.2 \pm 0.7$ & $2.6 \pm 0.5$ & $<0.01$ \\
\hline
\end{tabular}

Before, before the start of oral glucose tolerance test; OGTTih, value obtained at $1 \mathrm{~h}$ in the oral glucose tolerance test; SBP, systolic blood pressure; DBP, diastolic blood pressure; PRP, pressure rate products; $L F$, low-frequency power; $H F$, high-frequency power; $L F / H F$, ratio of $L F / H F$; TBARS, plasma level of thiobarbituric acid-reactive substances; CFVbase, mean diastolic coronary flow velocity at baseline; CFVhyp, mean diastolic coronary flow velocity during adenosine infusion; CFVR, coronary flow velocity reserve.
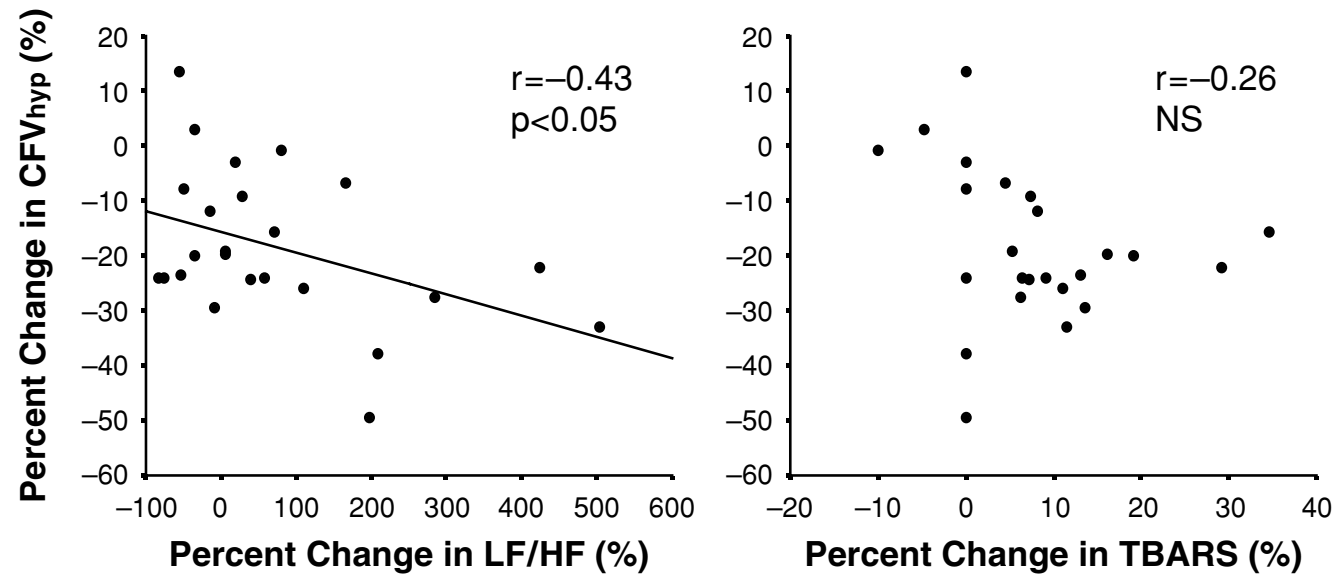

Fig 1. Relationship between the percent changes in the mean coronary blood flow velocity during adenosine infusion and the changes in the plasma levels of thiobarbituric acid-reactive substances and changes of the LF/HF induced by acute hyperglycemia. CVFhyp, mean coronary flow velocity following the infusion of adenosine; TBARS, plasma level of thiobarbituric acid-reactive substances: LF/HF, low/high frequency power.

cally significant.

\section{Results}

Table 1 shows the clinical characteristics of the subjects and Table 2 is a summary of the various parameters examined before and $1 \mathrm{~h}$ after the 75-g OGTT. AHG during the 75-g OGTT increased the heart rate, pressure-rate product, LF, LF/HF, TBARS, and CFVbase, and decreased CFVhyp and CFVR.

The results of simple linear regression analysis revealed that the percent change in $\mathrm{CFV}_{\text {hyp }}$ induced by AHG significantly correlated with the percent change in the LF/HF ratio, but not with the change in the level of TBARS (Fig 1). Stepwise multiple linear regression analysis demonstrated that the percent change in the CFVhyp caused by AHG correlated with the percent change in the $\mathrm{LF} / \mathrm{HF}$ ratio, even after adjustment for age, gender, smoking status, 75-g OGTT status and medications, as well as with the percent change in the level of TBARS, and of the pressure-rate product $(\mathrm{R}$-square $=0.18, \beta=-0.43, \mathrm{p}<0.05)$.

In addition, the relationship of the percent change in $\mathrm{LF} / \mathrm{HF}$ ratio caused by $\mathrm{AHG}$ with those in the sum of the plasma glucose levels during the 75-g OGTT (ie, before and 30,60 , and 120 min after ingestion of a solution containing $75 \mathrm{~g}$ of glucose) (SigmaBS), the sum of the plasma insulin levels (SigmaINS), and the percent change in the level of TBARS were assessed by multiple regression analysis. No significant association of the changes in LF/HF ratio induced by AHG with those of the other variables was observed.

\section{Discussion}

Hyperemic coronary blood flow response is one of the markers of coronary microvascular function ${ }^{21,22}$ and several studies have demonstrated that chronic hyperglycemia can reduce the increase in hyperemic coronary blood flow without changing the basal coronary blood flow! $12,23-25$ However, only a few studies have examined 
the effects of AHG on coronary microvascular function. Capaldo et al reported that AHG does not affect the coronary blood flow response to hyperemia in healthy subjects ${ }^{26}$ and Scognamiglio et al demonstrated that postprandial hyperglycemia increased the basal myocardial blood flow in healthy subjects but decreased it in subjects with type II diabetes mellitus; however, they did not examine the effects of postprandial hyperglycemia on the hyperemic coronary blood flow response.7 Although some studies have demonstrated that AHG impairs the acetylcholine- or reactive hyperemia-induced increase in the blood flow in peripheral arteries in subjects with atherosclerotic risk factors, 5,6 to the best of our knowledge, no study has examined the effects of AHG on coronary microvascular function in subjects with atherosclerotic risk factors. In our study, the subjects may be assumed to have had atherosclerotic arterial damage because they were receiving treatment for atherosclerotic risk factors, and AHG was associated with a decrease of the CFVhyp. Thus, AHG may impair coronary microvascular function, at least in subjects with a diseased coronary arterial bed.

Several mechanisms, including decreased NO bioavailability, increased oxidative stress, sympathetic activation, disturbances of intracellular signal transduction, and abnormal hemostasis, have been proposed to explain the coronary microvascular dysfunction associated with AHG5,6,28,29 In the present study, we focused on the influence of increased oxidative stress and sympathetic activation on the coronary microvascular dysfunction induced by AHG. Recent studies have suggested that an acute increase in oxidative stress may impair coronary microvascular function! 14,30 AHG directly increases the oxidative stress, $6,28,29,31,32$ and it is possible that this might contribute to the impairment of coronary microvascular function caused by AHG, Although isoprostan and 3-nitrotyrosine are established markers of oxidative stress in the clinical setting, $, 5,16$ these markers reflect steady-state oxidative stress and do not reflect any acute changes of oxidative stress. However, TBARS, a marker of lipid peroxidation, the levels of which change rapidly in response to changes in oxidative stress, are considered to be a reliable marker of acute changes in oxidative stress. In the present study, although AHG increased the level of TBARS, the percent change in the TBARS caused by AHG was not related to the percent change in the CFVhyp. Thus, the present study did not confirm any direct or close relationship between the impairment of coronary microvascular function and the increase in oxidative stress caused by AHG.

Cardiac autonomic dysfunction has been shown to be related to the development of diabetic cardiomyopathy 34 and to myocardial ischemia in subjects with diabetes mellitus 35 AHG activates sympathetic tone directly and/or via induction of hyperinsulinemia 36,37 In addition, increased oxidative stress also activates sympathetic tone ${ }^{38}$ In the present study, the results of multiple linear regression analysis demonstrated that the percent change in LF/HF, a marker of changes in sympathetic tone ${ }^{17-19}$ did not correlate with SigmaBS, SigmaINS, or the percent change in the level of TBARS. Therefore, AHG may induce sympathetic activation through multiple mechanisms, including hyperglycemia, hyperinsulinemia, and increased oxidative stress, all of which are also interrelated. The results of multiple linear regression analysis in this study revealed that the percent change in the LF/HF ratio was independently related to that in CFVhyp. Although sympathetic control of coronary vasomotor tone is thought not to be important in healthy subjects, it becomes functionally significant in subjects with endothelial dysfunction. In subjects such as those with atherosclerotic risk factors, increased sympathetic adrenergic tone may lead to coronary vasoconstriction with a concomitant increase in myocardial oxygen demand 39,40 The results of our present study suggest that the impairment of the coronary microvascular function induced by AHG might be directly related to activation of sympathetic tone.

CFVR has been also used in clinical experiments as a parameter to assess the effects of interventions on the coronary microcirculation.2-14 In those studies, CFV base was found to be unaffected by any of the interventions. On the other hand, in the present study AHG was associated with an increase of CFVbase and, therefore, evaluation of the changes in the CFVR might not be the most appropriate method of assessing the effect of interventions on the coronary microcirculation because CFVR is only a relative value (it is not based on an assessment of the absolute coronary blood flow) ${ }^{10-12}$

\section{Study Limitations}

The present study has the following limitations.

1. At least some of the subjects of this study might have had subclinical atherosclerosis. Further studies are needed to confirm the association among coronary microvascular dysfunction, increased oxidative stress, and sympathetic activation induced by AHG in healthy subjects and subjects with advanced atherosclerosis.

2. It is possible that the sympathetic activation induced by AHG also affects coronary vessel diameter. In addition, the deleterious effect of hyperglycemia on coronary vascular function might outweigh the coronary vasodilatation induced by the concomitant hyperinsulinemia; ${ }^{41}$ however, transthoracic echocardiography cannot be used to measure coronary vessel diameter. ${ }^{10-12}$ Transthoracic Doppler is not appropriate for measuring the absolute coronary blood flow ${ }^{10-12}$ and, therefore, confirmation of the present findings by direct measurements of the coronary blood flow would be the next logical step. In addition, an extra protocol to examine the effects of antioxidants, such as vitamin $\mathrm{C}$, on the impairment of coronary microvascular function caused by AHG is proposed.

\section{Conclusion}

Recent accumulated evidence suggests that not only chronic, but also acute, hyperglycemia is a direct and independent risk factor for cardiovascular disease and its outcome. In subjects with atherosclerotic risk factors who may be considered likely to have atherosclerotic arterial damage, AHG seems to concomitantly induce coronary microvascular dysfunction, increased oxidative stress, and sympathetic activation. Therefore, coronary microvascular dysfunction may be closely related to sympathetic activation. This phenomenon may contribute, at least in part, to the adverse cardiovascular outcomes reported in cases with postprandial hyperglycemia or stress hyperglycemia.

\section{References}

1. Meigs JB, Nathan DM, D'Agostino RB Sr, Wilson PW; Framingham Offspring Study. Fasting and postchallenge glycemia and cardiovascular disease risk: The Framingham Offspring Study. Diabetes Care 
2002; 25: $1845-1850$.

2. Capes SE, Hunt D, Malmberg K, Gerstein HC. Stress hyperglycaemia and increased risk of death after myocardial infarction in patients with and without diabetes: A systematic overview. Lancet 2000; 355: $773-778$.

3. Kosuge M, Kimura K, Kojima S, Sakamoto T, Matsui K, Ishihara M, et al; Japanese Acute Coronary Syndrome Study (JACSS) Investigators. Effects of glucose abnormalities on in-hospital outcome after coronary intervention for acute myocardial infarction. Circ J 2005; 69: $375-379$.

4. Ishii H, Ichimiya S, Kanashiro M, Amano T, Matsubara T, Murohara T. Effects of intravenous nicorandil before reperfusion for acute myocardial infarction in patients with stress hyperglycemia. Diabetes Care 2006; 29: 202-206.

5. Beckman JA, Goldfine AB, Gordon MB, Creager MA. Ascorbate restores endothelium-dependent vasodilation impaired by acute hyperglycemia in humans. Circulation 2001; 103: 1618-1623.

6. Kawano H, Motoyama T, Hirashima O, Hirai N, Miyao Y, Sakamoto $\mathrm{T}$, et al. Hyperglycemia rapidly suppresses flow-mediated endothelium-dependent vasodilation of brachial artery. $\mathrm{J} \mathrm{Am} \mathrm{Coll} \mathrm{Cardiol}$ 1999; 34: 146-154.

7. Brevetti G, Silvestro A, Schiano V, Chiariello M. Endothelial dysfunction and cardiovascular risk prediction in peripheral arterial disease: Additive value of flow-mediated dilation to ankle-brachial pressure index. Circulation 2003; 108: 2093-2098.

8. Kaufmann PA, Gnecchi-Ruscone T, di Terlizzi M, Schafers KP, Luscher TF, Camici PG. Coronary heart disease in smokers: Vitamin C restores coronary microcirculatory function. Circulation 2000; 102: $1233-1238$

9. Pop-Busui R, Kirkwood I, Schmid H, Marinescu V, Schroeder J, Larkin D, et al. Sympathetic dysfunction in type 1 diabetes: Association with impaired myocardial blood flow reserve and diastolic dysfunction. J Am Coll Cardiol 2004; 44: 2368-2374.

10. Dimitrow PP, Galderisi M, Rigo F. The non-invasive documentation of coronary microcirculation impairment: Role of transthoracic echocardiography. Cardiovasc Ultrasound 2005; 3: 18-19.

11. Hirata K, Amudha K, Elina R, Hozumi T, Yoshikawa J, Homma S, et al. Measurement of coronary vasomotor function: Getting to the heart of the matter in cardiovascular research. Clin Sci (Lond) 2004; 107: $449-460$.

12. Miyazaki C, Takeuchi M, Yoshitani H, Otani S, Sakamoto K, Yoshikawa J. Optimum hypoglycemic therapy can improve coronary flow velocity reserve in diabetic patients: Demonstration by transthoracic doppler echocardiography. Circ J 2003; 67: 945-950.

13. Hasegawa R, Daimon M, Toyoda T, Teramoto K, Sekine T, Kawata $\mathrm{T}$, et al. Effect of mental stress on coronary flow velocity reserve in healthy men. Am J Cardiol 2005; 96: 137-140.

14. Teramoto K, Daimon M, Hasegawa R, Toyoda T, Sekine T, Kawata $\mathrm{T}$, et al. Acute effect of oral vitamin $\mathrm{C}$ on coronary circulation in young healthy smokers. Am Heart J 2004; 148: 300-305.

15. Meagher EA, FitzGerald GA. Indices of lipid peroxidation in vivo: Strengths and limitations. Free Radic Biol Med 2000; 28: $1745-$ 1750 .

16. Cherubini A, Ruggiero C, Polidori MC, Mecocci P. Potential markers of oxidative stress in stroke. Free Radic Biol Med 2005; 39: $841-$ 852.

17. Nakao M, Nomura K, Karita K, Nishikitani M, Yano E. Relationship between brachial-ankle pulse wave velocity and heart rate variability in young Japanese men. Hypertens Res 2004; 27: 925-931.

18. Tateishi O, Shouda T, Azuma Y, Chin K, Nogimura T, Gotou Y, et al. Heart rate variability in congestive heart failure. Clin Exp Hypertens 2002; 24: 75-81.

19. Tomiyama H, Nakayama T, Watanabe G, Shiojima K, Sakuma Y, Yamamoto A, et al. Effects of short-acting and long-acting loop diuretics on heart rate variability in patients with chronic compensated congestive heart failure. Am Heart $J$ 1999; 137: 543-548.

20. World Health Organization. Definition, diagnosis and classification of diabetes mellitus and its complications: Report of WHO consultation. Part 1: Diagnosis and classification of diabetes mellitus. Geneva, WHO, 1999.

21. Smits SB, Williams DE, Lipson P, Banitt GA, Rongen Creager MA.
Endothelial release of nitric oxide contributes to the vasodilator effect of adenosine in humans. Circulation 1995; 92: 2135-2141.

22. Zeiher AM, Drexler H, Wollschläger H, Just H. Endothelial dysfunction of the coronary microvasculature is associated with impaired coronary blood flow regulation in patients with early atherosclerosis. Circulation 1991; 84: 1984-1992.

23. Yokoyama I, Ohtake T, Momomura S, Yonekura K, Woo-Soo S, Nishikawa $\mathbf{J}$, et al. Hyperglycemia rather than insulin resistance is related to reduced coronary flow reserve in NIDDM. Diabetes 1998; 47: $119-124$.

24. Prior JO, Quinones MJ, Hernandez-Pampaloni M, Facta AD, Schindler TH, Sayre JW, et al. Coronary circulatory dysfunction in insulin resistance, impaired glucose tolerance, and type 2 diabetes mellitus. Circulation 2005; 111: 2291-2298.

25. Di Carli MF, Janisse J, Grunberger G, Ager J. Role of chronic hyperglycemia in the pathogenesis of coronary microvascular dysfunction in diabetes. J Am Coll Cardiol 2003; 41: 1387-1393.

26. Capaldo B, Galderisi M, Turco AA, D'Errico A, Turco S, Rivellese AA, et al. Acute hyperglycemia does not affect the reactivity of coronary microcirculation in humans. J Clin Endocrinol Metab 2005; 90: $3871-3876$

27. Scognamiglio R, Negut C, De Kreutzenberg SV, Tiengo A, Avogaro A. Postprandial myocardial perfusion in healthy subjects and in type 2 diabetic patients. Circulation 2005; 112: 179-184.

28. Creager MA, Luscher TF, Cosentino F, Beckman JA. Diabetes and vascular disease: Pathophysiology, clinical consequences, and medical therapy: Part I. Circulation 2003; 108: 1527-1532.

29. Ceriello A. Impaired glucose tolerance and cardiovascular disease: The possible role of post-prandial hyperglycemia. Am Heart J 2004; 147: 803-807.

30. Yamashita K, Tasaki H, Nagai Y, Suzuka H, Nihei S, Kobayashi K, et al. Experimental hyperhomocysteinemia impairs coronary flow velocity reserve. Int J Cardiol 2005; 104: 163-169.

31. Monnier L, Mas E, Ginet C, Michel F, Villon L, Cristol JP, et al. Activation of oxidative stress by acute glucose fluctuations compared with sustained chronic hyperglycemia in patients with type 2 diabetes. JAMA 2006; 295: 1681-1687.

32. Marfella R, Quagliaro L, Nappo F, Ceriello A, Giugliano D. Acute hyperglycemia induces an oxidative stress in healthy subjects. J Clin Invest 2001; 108: 635-636.

33. Shinozaki K, Hirayama A, Nishio Y, Yoshida Y, Ohtani T, Okamura $\mathrm{T}$, et al. Coronary endothelial dysfunction in the insulin-resistant state is linked to abnormal pteridine metabolism and vascular oxidative stress. J Am Coll Cardiol 2001; 38: 1821 - 1828.

34. Fang ZY, Prins JB, Marwick TH. Diabetic cardiomyopathy: Evidence, mechanisms, and therapeutic implications. Endocr Re 2004; 25: $543-567$.

35. Wackers FJ, Young LH, Inzucchi SE, Chyun DA, Davey JA, Barrett EJ, et al; Detection of Ischemia in Asymptomatic Diabetics Investigators. Detection of silent myocardial ischemia in asymptomatic diabetic subjects: The DIAD study. Diabetes Care 2004; 27: 19541961.

36. Hoffman RP, Hausberg M, Sinkey CA, Anderson EA. Hyperglycemia without hyperinsulinemia produces both sympathetic neural activation and vasodilation in normal humans. J Diabetes Complications 1999; 13: 17-22.

37. Van De Borne P, Hausberg M, Hoffman RP, Mark AL, Anderson EA. Hyperinsulinemia produces cardiac vagal withdrawal and nonuniform sympathetic activation in normal subjects. Am J Physiol 1999; 276(1 Pt 2): R178-R183.

38. Ye S, Zhong H, Campese VM. Oxidative stress mediates the stimulation of sympathetic nerve activity in the phenol renal injury model of hypertension. Hypertension 2006; 48: 309-315.

39. Fiegel EO. Neural control of coronary blood flow. J Vasc Res 1998; 35: $85-92$.

40. Remme WJ. The sympathetic nervous system and ischaemic heart disease. Eur Heart J 1998; 19(Suppl F): F62-F71.

41. Srinivasan M, Herrero P, McGill JB, Bennik J, Heere B, Lesniak D, et al. The effects of plasma insulin and glucose on myocardial blood flow in patients with type 1 diabetes mellitus. $J$ Am Coll Cardiol 2005; 46: $42-48$. 